MATEC Web of Conferences 22,02010 (2015)

DOI: $10.1051 /$ matec conf/ 20152202010

(C) Owned by the authors, published by EDP Sciences, 2015

\title{
Research and Application of Auxiliary Optimization Technology of Power Grid Accident Processing Based on the Mode of Regulation and Control Integration
}

\author{
Houzhen Cui, Jun Qin, Dongya Wu \& Xiaoyong Du \\ State Grid Henan Electric Power Company, Zhengzhou, Henan, China
}

\begin{abstract}
Accident processing is the most important link of the scheduling of daily monitoring. The improvement of intelligent level is of great significance for improving the efficiency of accident processing scheduling, shortening the time of accident processing and preventing further deterioration of accidents. According to features of accident processing scheduling, this paper puts forward an integrated framework of aid decision-making of online accident processing based on large power grid, and carries out a study from five aspects, namely integrated information support platform, risk perception in advance, online fault diagnosis, aid decision-making afterwards and visual display, so as to conduct real-time tracking on operating state of power grid, eliminate potential safety hazards of power grid and upgrade power grid from "manual analysis" scheduling to "intelligent analysis" scheduling.
\end{abstract}

Keywords: integration of regulation and control; power grid accident; optimizing process

\section{INTRODUCTION}

With the constant reform and development of smart grid system, the traditional grid operation and management mode has been unable to satisfy the development requirements of current smart grid. Thus, it is obliged to reform the current scheduling and operation management system and distribution network management mode so as to establish a new system that is able to satisfy the development requirements of smart grid.

In recent years, the State Grid Corporation of China has formulated policies in accordance with development situations of grid companies of different provinces and cities so that the development of power grid becomes gradually interconnected and intelligent and the new situation of electric power resource allocation can be optimized and promoted in large scale. $\mathrm{Nu}$ merous problems need to be solved so as to prevent power outage of large areas caused by grid accidents. First, from the perspective of safe operation of power grid, it is necessary to collect real-time data of grid operating state and conduct analysis on the data so as to ensure the safe state of data and the reliable and safe operation of power grid. This is an important guarantee for the prevention of power outage of large areas caused by grid accidents. According to the development scale of power grid in Henan province, the current grid operation system structures and operation modes are relatively complicated, leading to various kinds of accidents to some extent, especially unavoidable accidents caused by unpredictable factors. Once there is an accident, huge hidden hazards will be brought to power grid if the accident cannot be han- dled timely and correctly. It is a new problem for the grid scheduling center of Henan province that what kind of measures should be taken to narrow the scope of power outage, shorten power outage time and prevent further expansion of accident area. This paper mainly proposes a study on the auxiliary optimization technology of power grid accident treatment based on the mode of regulation and control integration with the combination of the development status of power companies in Henan province.

\section{RESEARCH CONTENT OF THE AUXILIARY OPTIMIZATION TECHNOLOGY OF POWER GRID ACCIDENT PROCESSING BASED ON THE MODE OF REGULATION AND CON- TROL INTEGRATION}

Power grid accident analysis and processing are advanced applications of smart grid. Fault diagnosis is the core of grid accident analysis. At present, related researches at home and abroad are carried out in the following directions: 1) Fault information data extraction, including fault information types and features of diagnosis and pre-processing method of diagnosis. For example, fault diagnoses based on circuit breaker information and fault diagnoses based on circuit breaker and protection information are realized by SCADA system. The intelligent scheduling system of Lanzhou Power Supply Bureau first realizes fault diagnosis and recovery function by utilizing D5000 to make decisions and support power grid faults. 2) Studies on the diagnostic model, including reasoning diagnostic model and optimized diagnostic model. 
Reasoning diagnostic model is mainly the simulation and computer implementation of artificial diagnostic behaviors, a typical example of which is the expert database model. Models developed in late period include the diagnostic model of neural network, the diagnostic model based on Petri network, the diagnostic model based on the net of causation, and so on. Optimized diagnostic model finds out the optimal interpretation of alerting signals through an algorithm in line with the logical relationship between equipment and protective actions and breaker tripping. Optimization algorithms include genetic algorithm, simulated annealing algorithm, Tabu searching algorithm, particle swarm optimization, artificial fish swarm algorithm, and so on.

An integrated grid accident analysis system involves many aspects such as communication and data center. The auxiliary optimization system of power grid accident processing based on the mode of regulation and control integration consists of two parts, namely the auxiliary decision-making platform of accident processing and the integrated information support platform. The main content of the auxiliary optimization system of power grid accident processing-suggestions on fault diagnosis and analysis-is to optimize the safety of power grid. That is to say, static security data of power grid is analyzed when the grid is in safe mode so as to find out weak links of power grid and put forward preventive suggestions for adjustment.

\section{KEY TECHNOLOGIES}

Key technologies of auxiliary optimization system of power grid accident processing based on the mode of regulation and control integration mainly include three aspects, namely data information support platform, intelligent fault diagnosis and analysis technology, and aid decision-making of grid fault elimination (see Figure 1).

\subsection{Unified information platform based on IEC61850}

The basis of aid decision-making of accident processing is integrated information of power grid, including mainly grid model, operating data and analysis result. Therefore, a unified information platform should be established to provide unified data access and storage services for accident processing.

Grid operating data can be generally divided into three categories: operating data, fault data and PMU data. Operating data is collected and uploaded timely, mainly satisfying SCADA/EMS system requirements. Fault data refers to disturbance recording data, including fault recording and SOE.

IEC61850 provides a good mechanism to realize modeling and transmission of operating data. With RDRE, RADR and RBDR provided by IEC61850, effective association between fault recording information and SCD files can be realized through expansion so as to realize the semantic description of fault information. Establish a common format of IED event data and make event logging data (SOE, fault report, fault briefing) and be compatible in IEC61850 so as to provide sufficient semantic information for events.

Establish a basic system of integrated information of large power grid and realize the unified access of integrated information through virtual data access count so as to provide grid accidents with complete

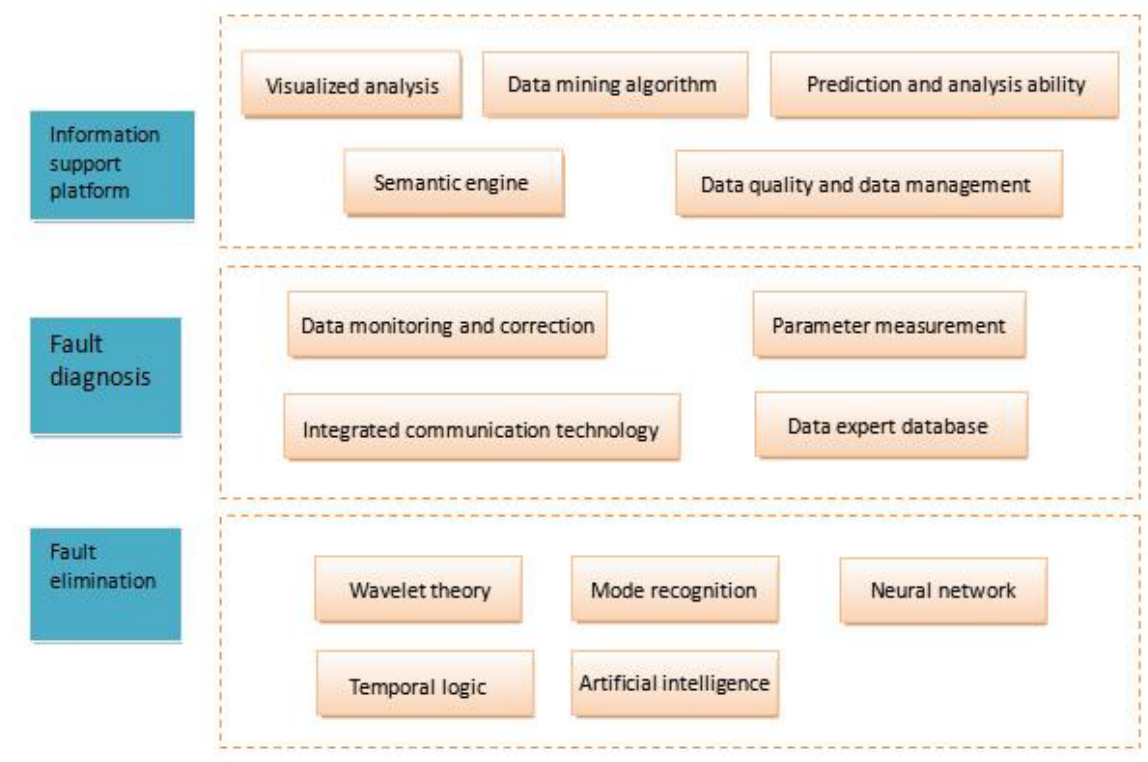

Figure 1. Key technologies of auxiliary optimization system of power grid accident processing based on the mode of regulation and control integration 
basic data.

\subsection{Function of intelligent fault diagnosis}

One of the major functions of the aid decision-making system of grid accident processing is the model of intelligent fault diagnosis and analysis, which is mainly in the service of scheduling personnel. It is required that fast and accurate warnings can be provided when the grid is in fault so as to emphasize the grid fault and the exact position, provide decision support for fault treatment scheduling and assist scheduling personnel to find the fault.

If a minority of grid components is in fault, relevant breakers will be cut off in a short time and components in fault will be separated from normal ones so as to prevent the expansion of accident. For this reason, fault components will be isolated in a passive network after the fault dies away. So, the variation of power outage scope caused by the fault can be determined through network topology analysis and the comparison of power outage area before and after the fault Solutions of a series of problems that cause accidents can be found through these changes.

Fault information platform integrates and associates operating data, fault recording files and event recording data together and conducts fault diagnosis with data of the fault information platform. Specifically speaking, fault recording files are able to record original waveforms of voltage and current completely in grid fault. Dynamic data records mutation information of voltage and current in grid fault. Steady state data records switch displacement and protection movement signals. Misjudgments caused by unreliable data can be reduced significantly according to comprehensive information judgment of state quantity (switch displacement, protection movement signals, etc.) and electrical quantity (mutation of voltage and current). As for instantaneity, preliminary judgment of fault can be carried out by taking full advantage of the feature of real-time upload of steady and dynamic data and then detailed analysis of fault can be conducted after the upload of data in transient state. As for comprehensiveness, preliminary judgment of separating and coinciding situations of fault equipment can be carried out with steady and dynamic data and then fault location and short-circuit current calculation can be further conducted in line with data in transient state, providing technical support for rapid master of grid faults in scheduling.

\subsection{Function of aid decision-making}

In accident processing of grid scheduling, the aid decision-making system is not only the core foundation of grid scheduling accident processing but also an important link of grid scheduling accident processing. In normal conditions, scheduling accident processing generally includes four links, namely fault recognition, emergency control, prevention control and fault re- covery processing. Fault recognition is to judge if there is a fault in grid scheduling and determine the exact position of the fault. Emergency control means to judge if there are mutations like over-ranging and super-flow current so that scheduling management personnel can process the accident immediately so as to prevent further expansion of accident area. Prevention control is to prevent over-ranging faults of new equipment after grid fault. Thus, further and deeper processing and inspection are required to avoid equipment over-ranging caused by secondary fault. Fault recovery processing means that the scheduling center conducts maintenance, inspection and elimination on faults.

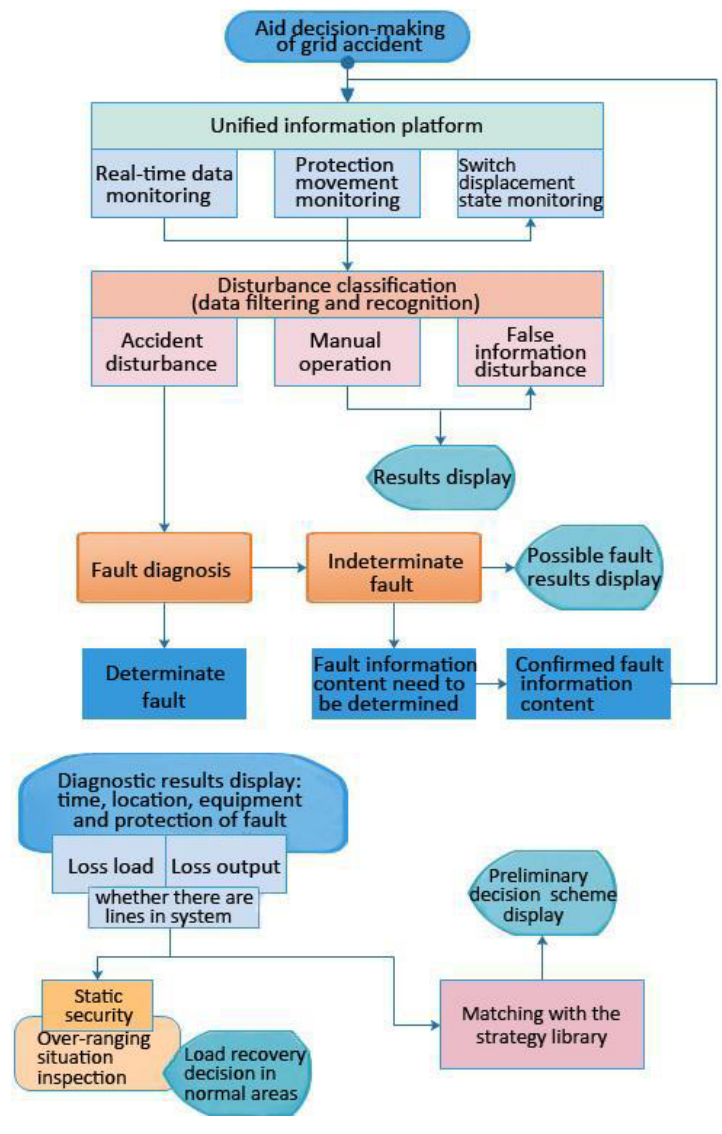

Figure 2.

4 RESEARCH AND APPLICATION SIGNIFICANCE OF THE AUXILIARY OPTIMIZATION TECHNOLOGY OF POWER GRID ACCIDENT PROCESSING BASED ON THE MODE OF REGULATION AND CONTROL INTEGRATION

The auxiliary optimization system of power grid accident processing based on the mode of regulation and control integration carries out real-time tracking on the 
operating state of power grid and eliminates safety hazards in power grid rapidly. On the one hand, the auxiliary optimization system of power grid accident processing will send out warning signals immediately once there is a fault in power grid so that scheduling management personnel can control the accident effectively and avoid the chain effect of subsequent accidents. On the other hand, the auxiliary optimization system of power grid accident processing based on the mode of regulation and control integration reduces the probability of grid accidents, increases the ability of spontaneous recovery and reduces contradictions like the loss of assets caused by grid accidents. The system not only provides a reference for the daily scheduling of management personnel but also helps scheduling personnel to make decisions, so that power grid can be upgraded from "manual analysis" scheduling to "intelligent analysis" scheduling.

At present, accident processing in the scheduling center mainly combines new changes, principle and methods of accident processing. The structural design and method of auxiliary optimization system of power grid accident processing based on the mode of regulation and control integration is proposed from the aspect of scheduling and monitoring personnel, realizing the transformation of scheduling operation from "streamlined information" to "intelligent prevention and control" as well as a great leap of grid scheduling from experience management to integrated management of intelligent analysis. The safe and reliable operation of power grid is not only the basis of the sound and steady development of the national economy but also the source that guarantees normal operation of each department. The power supply system is first influenced when there is an accident in power grid. Besides, a great economic loss will be brought to the whole society and the peaceful development of the nation will also be threatened.

\section{REFERENCES}

[1] Yu, Y.X. \& Luan, W.P. 2009. Intelligent power grid, Power System and Clean Energy, 25(1): 7-11.

[2] Yao, J.G., Yang, S.C., Gao, Z.H., et al. 2007. The development trend of the automatic system of grid scheduling, Automation of Electric Power Systems, 31(13): 7-11.

[3] Du, G.H. \& Wang, Z.F. 2010. Design and research of the integration of intelligent grid scheduling, Power System Protection and Control, 38(15): 127-131.

[4] Yang, S.C., Yao, J.G., Gao, Z.H., et al. 2006. A study on the aid decision-making of smart grid scheduling based on the secondary system of scheduling, Power System Technology, 30(supplement): 176-180.

[5] Yang, Y.H., Zhang, D.Y., Ma, Q., et al. 2004. A basic study on the security defense system of large grid, Power System Technology, 28(9): 23-27.

[6] Feng, S.G., Gao, R.B., Guo, X.Y. \& Liu, T. 2006. Research and implementation of the comprehensive auto- matic management information system of scheduling, Telecommunications for Electric Power System, 07. 